\title{
Linear versus nonlinear electro-optic effects in materials
}

\author{
Zhijun Jiang, ${ }^{1,2,3}$ Charles Paillard, ${ }^{2,4}$ Hongjun Xiang, ${ }^{1,5, *}$ and L. Bellaiche ${ }^{2, \dagger}$ \\ ${ }^{1}$ Key Laboratory of Computational Physical Sciences (Ministry of Education), \\ State Key Laboratory of Surface Physics, and Department of Physics, Fudan University, Shanghai 200433, China \\ ${ }^{2}$ Physics Department and Institute for Nanoscience and Engineering, \\ University of Arkansas, Fayetteville, Arkansas 72701, USA \\ ${ }^{3}$ School of Physics and Optoelectronic Engineering, Ludong University, Yantai 264025, China \\ ${ }^{4}$ Laboratoire SPMS, CentraleSupélec/CNRS UMR 8580, \\ Université Paris-Saclay, 8-10 rue Joliot Curie, 91190 Gif-sur-Yvette, France \\ ${ }^{5}$ Collaborative Innovation Center of Advanced Microstructures, Nanjing 210093, China
}

\begin{abstract}
Two schemes are proposed to compute the nonlinear electro-optic (EO) tensor for the first time. In the first scheme, we compute the linear EO tensor of the structure under a finite electric field, while we compute the refractive index of the structure under a finite electric field in the second scheme. Such schemes are applied to $\mathrm{Pb}(\mathrm{Zr}, \mathrm{Ti}) \mathrm{O}_{3}$ and $\mathrm{BaTiO}_{3}$ ferroelectric oxides. It is found to reproduce a recently observed feature, namely why $\mathrm{Pb}\left(\mathrm{Zr}_{0.52} \mathrm{Ti}_{0.48}\right) \mathrm{O}_{3}$ adopts a mostly linear $\mathrm{EO}$ response while $\mathrm{BaTiO}_{3}$ exhibits a strongly nonlinear conversion between electric and optical properties. Furthermore, the atomistic insight provided by the proposed $a b$-initio scheme reveals the origin of such qualitatively different responses, in terms of the field-induced behavior of the frequencies of some phonon modes and of some force constants.
\end{abstract}

Most materials exhibit a change in their refractive index when under applied static or low-frequency electric fields, a phenomenon known as the electro-optic (EO) effect $[1,2]$ and which is promising for some technologies [3-6]. In particular, having large nonlinear electro-optic coefficients would open the door for the design of novel devices [7-16]. For instance, it is important for EO modulation [13], high-speed optical shutters [14], electro-optical detection [15], and electrooptical switching [16]. Understanding at an atomistic level linear versus nonlinear EO effects should also be of large fundamental interest. For instance, it should resolve the current mystery of why a recent experiment [5] observed, in the $\mathrm{THz}$ regime, a linear electro-optic coefficient in $\mathrm{Pb}(\mathrm{Zr}, \mathrm{Ti}) \mathrm{O}_{3}$ while $\mathrm{BaTiO}_{3}$ films rather exhibit significant nonlinear (secondorder) electro-optic coefficients.

In view of such facts, having a first-principles-based technique allowing the computation of nonlinear conversion between electric and optical quantities but also providing a deep atomistic insight of such conversion is highly desired. However, such technique and ab-initio capabilities do not presently exist.

The aims of this paper are to demonstrate that such technique (1) can, in fact, be easily developed and applied to any material; (2) reproduces the experimental finding about the different nature (i.e., linear versus nonlinear) of the EO response of $\mathrm{Pb}(\mathrm{Zr}, \mathrm{Ti}) \mathrm{O}_{3}$ versus $\mathrm{BaTiO}_{3}$; and (3) explains such difference, via the field-induced behavior of some specific phonon frequencies and of the force constants of some chemical bonds.

Here, we employed the ABINIT package [17] with the local density approximation (LDA) to the density functional theory (DFT) and norm-conserving pseudopotentials [18], chosen in part to facilitate the computation of electro-optic coefficients $[4,19]$. The alchemical mixing approximation implemented in the ABINIT package [20], which is the pseudopotentials specific implementation of the virtual crystal approximation, is also adopted to investigate the $\mathrm{Pb}\left(\mathrm{Zr}_{0.52} \mathrm{Ti}_{0.48}\right) \mathrm{O}_{3}$ (PZT) solid solutions. We use a $8 \times 8 \times 8$ grid of special $\mathbf{k}$ points and a plane-wave kinetic energy cutoff of 50 hartree. The effects of $d c$ electric fields applied along the [111] direction on structural properties of the rhombohedral $R 3 \mathrm{~m}$ phase of both $\mathrm{BaTiO}_{3}$ (BTO) and PZT are simulated by taking advantage of the method developed in Refs. [21-24] (note that the R3m phase is the well-known ground state of BTO, and that we chose to study a Ti composition of $48 \%$ in PZT in order to have a stable rhombohedral ferroelectric state as well). Technically, for each considered magnitude of the $d c$ electric field, both the lattice parameters and the atomic positions were fully relaxed until the force acting on each atom is smaller than $5 \times$ $10^{-5}$ hartree/bohr. The resulting field-induced structures are then used as input for the ab-initio method of Refs. [4, 19, 25], that is based on the linear response of the optical dielectric tensor induced by a static (or low frequency) electric field $E_{k}$ and that has been proven to accurately compute EO coefficients in ferroelectric oxides (note that no electric field is incorporated when employing this latter method on the field-induced structures). Such coefficients obey the following equation

$$
\Delta\left(\varepsilon^{-1}\right)_{i j}=\sum_{k=1}^{3} \mathcal{R}_{i j k}^{\eta} E_{k}
$$

where $\left(\varepsilon^{-1}\right)_{i j}$ is the inverse of the electronic dielectric tensor that depends on the electric field. It is important to realize that, in our case, $\mathcal{R}_{i j k}^{\eta}$ is a clamped (strain-free) EO tensor that can practically depend on $E_{k}$ since we used the crystal structure spanned by this electric field for its calculation. In particular, plotting $\mathcal{R}_{i j k}^{\eta}$ versus $E_{k}$ will naturally determine if the materials under investigation only adopt a linear EO effect (in that case, $\mathcal{R}_{i j k}^{\eta}$ will be independent of $E_{k}$ ) or rather a nonlinear conversion between electric and optical quantities (which will make $\mathcal{R}_{i j k}^{\eta}$ dependent on $E_{k}$ ).

As detailed in Refs. [4, 19], $\mathcal{R}_{i j k}^{\eta}$ can be expressed as the sum of two contributions: a bare electronic part, $\mathcal{R}_{i j k}^{\mathrm{el}}$, which 
is proportional to the nonlinear optical dielectric susceptibility $\chi_{i j k}^{(2)}$, and an ionic part, $\mathcal{R}_{i j k}^{\mathrm{ion}}$, which is caused by the relaxation of the atomic positions due to the applied electric field and which depends on the first-order change of the linear dielectric susceptibility. The origin of the ionic contribution is related to the Raman susceptibility $\alpha_{i j}^{m}$ of mode $m$, the transverse optic mode polarity $p_{k}^{m}$ and phonon mode frequencies $\omega_{m}$. The clamped (strain-free) EO tensor is thus given by:

$\mathcal{R}_{i j k}^{\eta}=\mathcal{R}_{i j k}^{\mathrm{el}}+\mathcal{R}_{i j k}^{\mathrm{ion}}=\frac{-8 \pi}{n_{i}^{2} n_{j}^{2}} \chi_{i j k}^{(2)}-\frac{4 \pi}{n_{i}^{2} n_{j}^{2} \sqrt{\Omega_{0}}} \sum_{m} \frac{\alpha_{i j}^{m} p_{k}^{m}}{\omega_{m}^{2}}$,

where $n_{i}$ and $n_{j}$ are the principal refractive indices, and $\Omega_{0}$ is the unit cell volume. As taken advantage in previous works [4, 6, 19, 25], Eq. (2) can be used to provide a deep insight into EO coefficients. Examples include the determination of the modes $m$ mostly responsible for the value of these coefficients as well as their enhancement via the softening of these modes (i.e., $\omega_{m}$ approaching a zero value). Note also that there is an unclamped (stress-free that adds a contribution involving elasto-optic effects and piezoelectricity to the clamped one) EO tensor, that is given by $\mathcal{R}_{i j k}^{\sigma}=\mathcal{R}_{i j k}^{\eta}+\sum_{\alpha, \beta=1}^{3} p_{i j \alpha \beta} d_{k \alpha \beta}$ where $p_{i j \alpha \beta}$ are elasto-optic coefficients and $d_{k \alpha \beta}$ are piezoelectric strain coefficients $[4,6,19,25]$, but that we numerically found (see Fig. S1 of the Supplemental Material (SM) [26]) that $\mathcal{R}_{i j k}^{\sigma}$ and $\mathcal{R}_{i j k}^{\eta}$ behave in a similar qualitative and even quantitative way with the applied electric field, in both PZT and BTO. Consequently, we focus here on $\mathcal{R}_{i j k}^{\eta}$. Note also that, as detailed in the SM [26], we also computed the EO tensor associated with the aforementioned fieldinduced structures by using another (more brute force) method - since we are not aware that nonlinear electro-optic effects have ever been investigated using first-principles-based calculations. This latter method and the one explained above provide very similar results, which therefore attests of the validity of the approach adopted in this manuscript.

Let us now choose the Cartesian axes such as the $z$-axis is along the [111] polarization pseudo-cubic direction and the $y$-axis is perpendicular to the mirror plane of the $R 3 m$ structure, for both PZT and BTO. With this choice of axes and when adopting the Voigt notation, the $\mathcal{R}_{i j k}^{\eta}$ EO tensor has four independent elements: $\mathcal{R}_{11}^{\eta}, \mathcal{R}_{13}^{\eta}, \mathcal{R}_{33}^{\eta}$ and $\mathcal{R}_{51}^{\eta}$. Figures 1(a) and 1(b) show all these components as a function of the electric field applied along the [111] direction in PZT and BTO, respectively, as calculated from Eq. (2). Regarding PZT, Fig. 1(a) indicates that the clamped EO coefficients are predicted to be, at zero electric field and by order of increasing strength, $\mathcal{R}_{11}^{\eta}=-4.9 \mathrm{pm} / \mathrm{V}, \mathcal{R}_{13}^{\eta}=5.6 \mathrm{pm} / \mathrm{V}, \mathcal{R}_{33}^{\eta}=11.9$ $\mathrm{pm} / \mathrm{V}$ and $\mathcal{R}_{51}^{\eta}=12.6 \mathrm{pm} / \mathrm{V}$ (note that we are not aware of any measurement or calculation of these coefficients in rhombohedral PZT). When the electric field is turned on and increases in PZT, $\mathcal{R}_{33}^{\eta}$ quickly becomes the largest element and basically only very slightly linearly decreases, therefore indicating the occurrence of a predominant linear EO coefficient accompanied by a weak second-order EO response. In fact, one can nicely fit $\mathcal{R}_{33}^{\eta}$ by $r_{33}^{\eta}+s_{333}^{\eta} E_{3}$, which provides
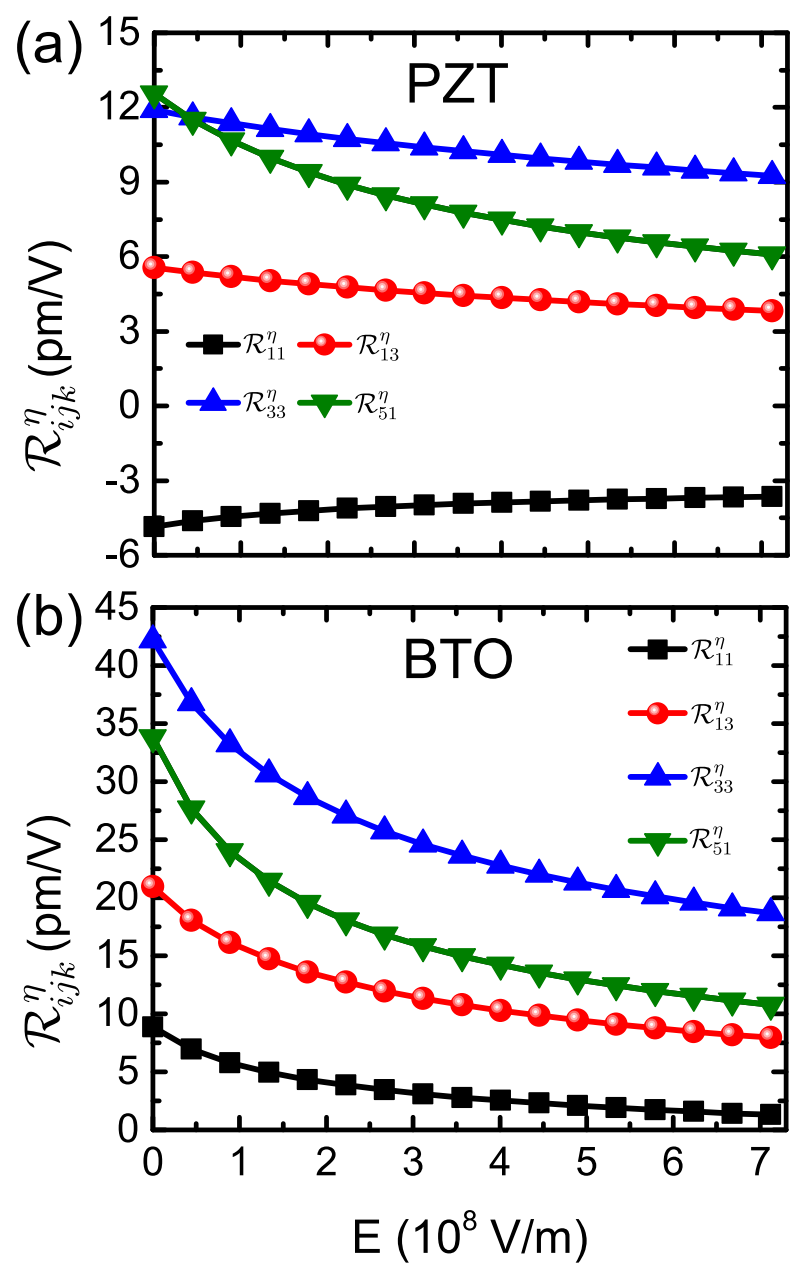

FIG. 1. Clamped EO coefficients as a function of electric field applied along the [111] direction in (a) PZT and (b) BTO, respectively.

a linear (Pockels effect) EO coefficient of $r_{33}^{\eta}=11.6 \mathrm{pm} / \mathrm{V}$ and a quadratic (Kerr effect) EO parameter of $s_{333}^{\eta}=-3.6 \times$ $10^{-21} \mathrm{~m}^{2} / \mathrm{V}^{2}$. Our predicted $r_{33}^{\eta}$ at $0 \mathrm{~K}$ is of the same order of magnitude and rather consistent with the measurement of Ref. [5] giving a value of $67.3 \mathrm{pm} / \mathrm{V}$ at room temperature for $\mathrm{Pb}\left(\mathrm{Zr}_{0.52} \mathrm{Ti}_{0.48}\right) \mathrm{O}_{3}$ at an applied $a c$ frequency of $1 \mathrm{THz}$, when realizing that temperature decreases the soft-mode frequencies and therefore enhances EO coefficient - as clearly indicated by Eq. (2).

For BTO at zero field, all the clamped EO coefficients are larger in magnitude than those of PZT, as shown in Fig. 1(b) that reports a value of $\mathcal{R}_{11}^{\eta}=8.9 \mathrm{pm} / \mathrm{V}, \mathcal{R}_{13}^{\eta}=21.0 \mathrm{pm} / \mathrm{V}$, $\mathcal{R}_{33}^{\eta}=42.2 \mathrm{pm} / \mathrm{V}$ and $\mathcal{R}_{51}^{\eta}=33.8 \mathrm{pm} / \mathrm{V}$ (note that these values are consistent with the previously reported ones of Ref. [19] but using the experimental lattice constants of BTO). Moreover and in sharp contrast with PZT, Fig. 1(b) also reveals that all the elements of $\mathcal{R}_{i j k}^{\eta}$ in BTO strongly depends on the magnitude of the electric field. Such numerical finding is fully in-line with a recent experiment [5] observing a predominantly linear EO response in PZT films versus a non- 
linear electro-optic response of BTO thin films, in the $\mathrm{THz}$ frequency range. In fact, we numerically find that our computed $\mathcal{R}_{33}^{\eta}$ of BTO of Fig. 1(b) can be very well fitted by $r_{33}^{\eta}+s_{333}^{\eta} E_{3}+c_{3333}^{\eta} E_{3}^{2}$, with $r_{33}^{\eta}=39.6 \mathrm{pm} / \mathrm{V}, s_{333}^{\eta}=-6.4 \times$ $10^{-20} \mathrm{~m}^{2} / \mathrm{V}^{2}$ and $c_{3333}^{\eta}=5.1 \times 10^{-29} \mathrm{~m}^{3} / \mathrm{V}^{3}$. Note, however, that the magnitude of the predicted second-order EO coefficient of $6.4 \times 10^{-20} \mathrm{~m}^{2} / \mathrm{V}^{2}$ is about 200 times smaller in magnitude than that measured in Ref. [5] for an $a c$ frequency of $1 \mathrm{THz}$ (note that the other method described in the SM [26] does not rely on linear response and provides similar result for the EO coefficients). Possible reasons behind such discrepancy is that we study here the $R 3 m$ phase at $0 \mathrm{~K}$ while experiments are conducted on the tetragonal phase of BTO at room temperature, that is very close (namely by about 20 $\mathrm{K}[2,31,32])$ to the tetragonal-to-orthorhombic phase transition where a large enhancement of the EO responses is expected [33] due to the softening of some phonon frequencies - as evidenced by Eq. (2). Other possible reasons may be that ab-initio electric fields can typically provide an overestimation by one or two orders of magnitude with respect to experimental ones [34-37], or that the experiment in Ref. [5] is conducted on strained and unpoled samples while we study bulk polar materials. The latter such hypothesis is even more reasonable when assuming the formula indicated in Refs. [7, 11, 33], that are: $\mathcal{R}_{13}^{0}=\frac{2}{3}\left(g_{11}+2 g_{12}-g_{44}\right) P_{s} \chi_{33}$, $\mathcal{R}_{33}^{0}=\frac{2}{3}\left(g_{11}+2 g_{12}+2 g_{44}\right) P_{s} \chi_{33}$, and $\mathcal{R}_{51}^{0}=$ $\frac{2}{3}\left(g_{11}-g_{12}+\frac{1}{2} g_{44}\right) P_{s} \chi_{11}$, where $\mathcal{R}_{i j}^{0}$ are linear EO coefficients, $P_{s}$ is the spontaneous polarization, $\chi_{33}$ and $\chi_{11}$ are the dielectric constants along the $c$ - and $a$-axes, respectively, and $g_{i j}$ are specific quadratic EO coefficients - all under infinitesimally small electric fields. As a matter of fact, plugging our numerical values for $\mathcal{R}_{i j}^{0}, P_{s}, \chi_{33}$ and $\chi_{11}$ into these formula gives for the $R 3 \mathrm{~m}$ phase of BTO bulk: $g_{11}=273, g_{12}=-2$ and $g_{44}=68$ in units of $10^{-2} \mathrm{~m}^{4} / \mathrm{C}^{2}$, which is precisely the order of magnitude reported in Ref. [7] for the $g_{i j}$ coefficients of BTO bulk at room temperature (i.e., in the $P 4 \mathrm{~mm}$ phase).

In order to understand the origin of the linear electro-optic response in PZT versus the nonlinear EO behavior in BTO, we determined the contribution of each zone-center phonon mode for the $\mathcal{R}_{33}^{\eta}$ coefficient, as well as the density functional perturbation theory (DFPT)-predicted frequency of these modes (see Fig. S3 of the SM [26] for all these zone-center phonons), for each of these two systems and for each investigated electric field. For PZT, Fig. 2(a) reveals that $\mathcal{R}_{33}^{\eta}$ mostly arises from the $A_{1}^{(1)}$ and especially $A_{1}^{(3)}$ modes, with these two modes having frequencies [see Fig. 2(c)] behaving in such a manner that the $\omega^{-2}$ inverse of their square is only weakly (and nearly linearly dependent) on the applied electric field as evidenced in Fig. 2(e). Incorporating such latter fact when looking again at Eq. (2) naturally explains why $\mathcal{R}_{33}^{\eta}$ is mostly independent of the electric field, that is why the EO response of PZT system is basically linear (with the slight change of $\omega^{-2}$ with field generating a weak second-order EO response). Note that the electronic part of the clamped EO tensor (first term of Eq. (2)) is found to be small, as revealed by Fig. 2(a)
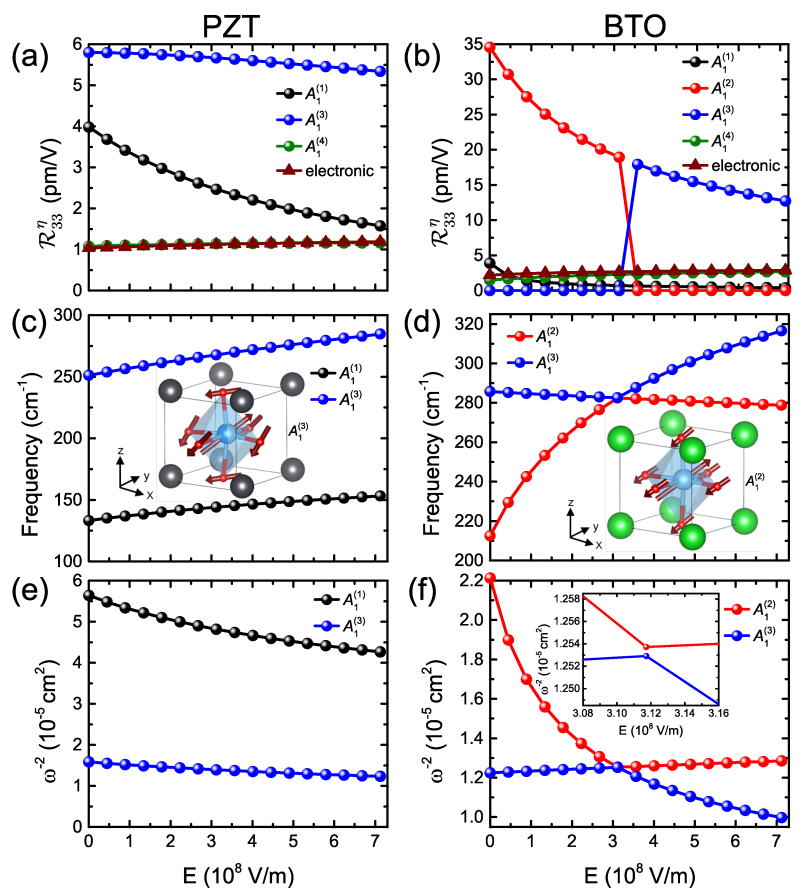

FIG. 2. Mode decomposition of the clamped EO coefficient $\mathcal{R}_{33}^{\eta}$ in (a) PZT and (b) BTO. Phonon frequency for selected modes at the $\Gamma$ point of the first Brillouin zone with the insets corresponding to the eigenvector of $A_{1}^{(3)}$ and $A_{1}^{(2)}$ modes at zero field in (c) PZT and (d) BTO, respectively. The inverse of the square of the phonon frequency, $\omega^{-2}$, as a function of electric field in (e) PZT and (f) BTO. The inset of panel (f) zooms in the data for electric field between 3.08 $\times 10^{8} \mathrm{~V} / \mathrm{m}$ and $3.16 \times 10^{8} \mathrm{~V} / \mathrm{m}$.

- therefore indicating the predominant role of ionic contributions for $\mathcal{R}_{33}^{\eta}$ of PZT [the same finding holds for BTO, as demonstrated in Fig. 2(b)]. Note also that, as indicated in the inset of Fig. 2(c), the Ti/Zr ions move along the spontaneous polarization [111] pseudo-cubic direction while the three oxygen ions move along the $[\overline{1} \overline{1} 0],[\overline{1} 0 \overline{1}]$ and $[0 \overline{1} \overline{1}]$ directions, respectively, in the $A_{1}^{(3)}$ mode of PZT.

In contrast, for BTO, $\mathcal{R}_{33}^{\eta}$ takes most of its value from the $A_{1}^{(2)}$ mode for fields smaller than $\simeq 3.1 \times 10^{8} \mathrm{~V} / \mathrm{m}$, with this mode having a frequency strongly increasing, and thus an inverse of the square of such frequency strongly decreasing, with the field. Consequently and according to Eq. (2) also, the electro-optic response of BTO is highly nonlinear, and is significantly reduced, for fields smaller than $\simeq 3.1 \times$ $10^{8} \mathrm{~V} / \mathrm{m}$. Note also that the nonlinear behavior of $\omega^{-2}$ with field is the culprit behind the existence of third-order EO coefficient. The inset of Fig. 2(d) shows that Ti ions move along the [111] direction while the $\mathrm{O}$ ions are displaced along the [1]1̄1] direction in the $A_{1}^{(2)}$ mode. Furthermore and as evidenced in Figs. 2(d) and 2(f), a striking anticrossing between the $A_{1}^{(2)}$ and $A_{1}^{(3)}$ modes then occurs in BTO for a field equal to $\simeq 3.1 \times 10^{8} \mathrm{~V} / \mathrm{m}$. Such anticrossing results in these modes repelling each other near this critical field, therefore yielding a gap between these two phonon frequencies and thus be- 

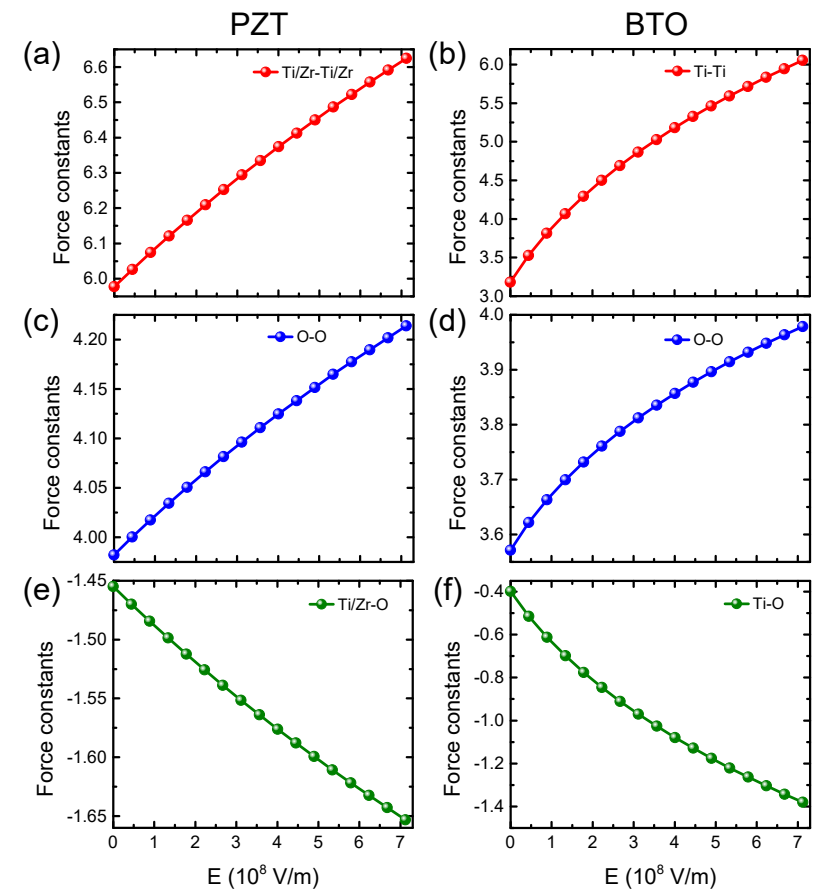

FIG. 3. Force constants at the $\Gamma$ point of the first Brillouin zone as a function of electric field for (a) Ti/Zr-Ti/Zr, (c) O-O and (e) Ti/Zr-O bonds in PZT; and (b) Ti-Ti, (d) O-O, and (f) Ti-O bonds in BTO.

tween the inverse of their square, as clearly seen in the inset of Fig. 2(f). This anticrossing also leads to the eigenvectors of these two modes inverting their atomic character before versus after this critical field [25]. It also makes the $A_{1}^{(3)}$ mode the dominant one for $\mathcal{R}_{33}^{\eta}$ above this critical field, with the resulting EO response being still nonlinear - since the frequency of the $A_{1}^{(3)}$ mode also strongly depends on the magnitude of the field above $\simeq 3.1 \times 10^{8} \mathrm{~V} / \mathrm{m}$.

One can thus safely conclude that the linear character of the EO response in PZT versus the nonlinear electro-optic response of BTO mainly originate from the different behavior that the $\omega^{-2}$ of their corresponding predominant modes adopt in these two important perovskite oxides.

The next issue to address is therefore to understand why these $\omega^{-2}$ behave in a different manner in PZT and BTO. For that, we reported the electric-field dependence of the force constants $\mathrm{Ti} / \mathrm{Zr}-\mathrm{Ti} / \mathrm{Zr}$, O-O and Ti/Zr-O bonds of PZT in the left column of Fig. 3, and of the force constants of Ti-Ti, O-O and Ti-O of BTO in the the right column of Fig. 3. The choice to concentrate on these specific force constants (rather than those involving $\mathrm{Pb}$ or $\mathrm{Ba}$ ions) stems from the atomic character of the eigenvectors associated with the $A_{1}^{(3)}$ mode in PZT and the $A_{1}^{(2)}$ mode in BTO [see again the inset of Figs. 2(c) and 2(d), respectively]. The selected force constants of Fig. 3 show nearly linear versus strongly nonlinear behaviors as a function of electric field in PZT and BTO, respectively, which therefore connects (and explains) the different nature of the macroscopic electro-optic response in these two systems to some specific atomistic bond characteristics.
In summary, a first-principle technique is developed to tackle nonlinear electro-optic response of materials at an $a b$ initio level for the first time, to the best of our knowledge. This method simply consists of first employing the development of Ref. [38] to determine the crystal and atomic structure induced by electric fields and then use such structure as input of the method of Refs. [4, 19] to extract EO coefficients as a function of electric field (note that other atomistic methods, such as those of Refs. [39, 40], can be used to extract the fieldinduced structure). This method is presently applied to the $R 3 m$ phase of PZT and BTO ferroelectric perovskite oxides, and is also found to provide similar results than another, more brute-force technique further proposed and explained in the SM [26]. Both of these methods reproduce a recent striking experimental finding, that is why the EO response of PZT and BTO is linear versus nonlinear, respectively [5] (note that the SM [26] also shows that other optical properties can behave in a different qualitative way between these two important materials). The scheme indicated in this manuscript also naturally reveals that it is the field-induced behavior of the frequency of some specific phonon modes and of some force constants that are responsible for the difference in nature for the conversion between electric and optical properties in PZT and BTO. We thus hope that the present study enhances the knowledge of light-matter interactions and functional materials, and will also motivate the development of other techniques allowing the investigation of complex interplay between light and physical properties. A particular advantage of the proposed method is that it can be easily employed for the quest of materials with large nonlinear EO response.

This work is supported by the National Natural Science Foundation of China (Grants No. 11804138 and No. 11825403), Shandong Provincial Natural Science Foundation (Grant No. ZR2019QA008), China Postdoctoral Science Foundation (Grant No. 2018M641905), the Qing Nian Ba Jian Program, Postdoctoral International Exchange Program of Academic Exchange Project, Fudan University Super Postdoctoral Program, and Shanghai Post-doctoral Excellence Program. C.P. and L.B. thank the ARO Grant No. W911NF-16-1-0227. L.B. also acknowledges the DARPA grant HR0011-15-2-0038 (MATRIX program).

* hxiang@ fudan.edu.cn

† laurent@uark.edu

[1] M. J. Weber, Handbook of Optical Materials (CRC Press, Boca Raton, FL, 2002).

[2] M. E. Lines and A. M. Glass, Principles and Applications of Ferroelectrics and Related Materials (Oxford University Press, New York, 1977).

[3] M. Zgonik, P. Bernasconi, M. Duelli, R. Schlesser, P. Günter, M. H. Garrett, D. Rytz, Y. Zhu, and X. Wu, Phys. Rev. B 50, 5941 (1994).

[4] M. Veithen, X. Gonze, and Ph. Ghosez, Phys. Rev. Lett. 93, 187401 (2004).

[5] L. Chen, Y. Zhang, Q. Guo, D. Zhang, X. Zhong, and J. Yuan, 
Appl. Phys. Lett. 105, 112903 (2014).

[6] C. Paillard, S. Prokhorenko, and L. Bellaiche, npj Comput. Mater. 5, 6 (2019).

[7] S. H. Wemple and M. DiDomenico, Jr., Appl. Solid State Sci. 3, 263 (1972).

[8] B. E. A. Saleh and M. C. Teich, Fundamentals of Photonics (Wiley, New York, 1991).

[9] A. Yariv and P. Yeh, Photonics: Optical Electronics in Modern Communications (Oxford University Press, New York, 2007).

[10] R. W. Boyd, Nonlinear Optics, 3rd ed. (Academic Press, New York, 2008).

[11] M. DiDomenico, Jr. and S. H. Wemple, J. Appl. Phys. 40, 720 (1969).

[12] S. H. Wemple and M. DiDomenico, Jr., J. Appl. Phys. 40, 735 (1969).

[13] M. G. Kuzyk, J. E. Sohn, and C. W. Dirk, J. Opt. Soc. Am. B 7, 842 (1990).

[14] Y. Hisakado, H. Kikuchi, T. Nagamura, and T. Kajiyama, Adv. Mater. 17, 96 (2005).

[15] R. L. Jin, Y. H. Yu, H. Yang, F. Zhu, Q. D. Chen, M. B. Yi, and H. B. Sun, Opt. Lett. 37, 842 (2012).

[16] T. Z. Shen, S. H. Hong, and J. K. Song, Nat. Mater. 13, 394 (2014).

[17] X. Gonze, J.-M. Beuken, R. Caracas, F. Detraux, M. Fuchs, G.M. Rignanese, L. Sindic, M. Verstraete, G. Zerah, F. Jollet, M. Torrent, A. Roy, M. Mikami, Ph. Ghosez, J.-Y. Raty, and D. C. Allan, Comput. Mater. Sci. 25, 478 (2002).

[18] D. R. Hamann, Phys. Rev. B 88, 085117 (2013).

[19] M. Veithen, X. Gonze, and Ph. Ghosez, Phys. Rev. B 71, 125107 (2005).

[20] P. Scharoch, M. J. Winiarski, and M. P. Polak, Comput. Mater. Sci. 81, 358 (2014).

[21] R. W. Nunes and D. Vanderbilt, Phys. Rev. Lett. 73, 712 (1994).

[22] R. W. Nunes and X. Gonze, Phys. Rev. B 63, 155107 (2001).

[23] I. Souza, J. Íñiguez, and D. Vanderbilt, Phys. Rev. Lett. 89, 117602 (2002).

[24] J. W. Zwanziger, J. Galbraith, Y. Kipouros, M. Torrent, M. Gi- antomassi, X. Gonze, Comput. Mater. Sci. 58, 113 (2012).

[25] Z. Jiang, C. Paillard, D. Vanderbilt, H. Xiang, and L. Bellaiche, Phys. Rev. Lett. 123, 096801 (2019).

[26] See Supplemental Material at [URL will be inserted by publisher] for more details about (i) clamped and unclamped electro-optic (EO) coefficients; (ii) another possible ab-initio way to compute EO coefficients for systems under electric field, and its comparison with the one described in the manuscript; (iii) all the zone-center phonon frequencies; and (iv) the electronic band gap of $\mathrm{Pb}\left(\mathrm{Zr}_{0.52} \mathrm{Ti}_{0.48}\right) \mathrm{O}_{3}$ (PZT) and $\mathrm{BaTiO}_{3}$ (BTO), which includes Refs. [27-30].

[27] S. Cabuk, Cent. Eur. J. Phys. 10, 239 (2012).

[28] M. Gajdoš, K. Hummer, G. Kresse, J. Furthmüller, and F. Bechstedt, Phys. Rev. B 73, 045112 (2006).

[29] S. Sharma and C. Ambrosch-Draxl, Phys. Scr. T109, 128 (2004).

[30] L. Chen, Y. Yang, Z. Gui, D. Sando, M. Bibes, X. K. Meng, and L. Bellaiche, Phys. Rev. Lett. 115, 267602 (2015).

[31] G. H. Kwei, A. C. Lawson, S. J. L. Billinge, and S.-W. Cheong, J. Phys. Chem. 97, 2368 (1993).

[32] L. Walizer, S. Lisenkov, and L. Bellaiche, Phys. Rev. B 73, 144105 (2006).

[33] M. Veithen and Ph. Ghosez, Phys. Rev. B 71, 132101 (2005).

[34] B. Xu, J. Íñiguez, and L. Bellaiche, Nat. Commun. 8, 15682 (2017).

[35] Z. Jiang, Y. Nahas, S. Prokhorenko, S. Prosandeev, D. Wang, J. Íñiguez, and L. Bellaiche, Phys. Rev. B 97, 104110 (2018).

[36] J. Lu, G. Chen, W. Luo, J. Íñiguez, L. Bellaiche, and H. Xiang, Phys. Rev. Lett. 122, 227601 (2019).

[37] L. Chen, C. Xu, H. Tian, H. Xiang, J. Íñiguez, Y. Yang, and L. Bellaiche, Phys. Rev. Lett. 122, 247701 (2019).

[38] H. Fu and L. Bellaiche, Phys. Rev. Lett. 91, 057601 (2003).

[39] P. Umari and A. Pasquarello, Phys. Rev. Lett. 89, 157602 (2002).

[40] N. Sai, K. M. Rabe, and D. Vanderbilt, Phys. Rev. B 66, 104108 (2002). 